\title{
Die sportmedizinische Mannschaftsbetreuung - ein rechtliches Minenfeld?
}

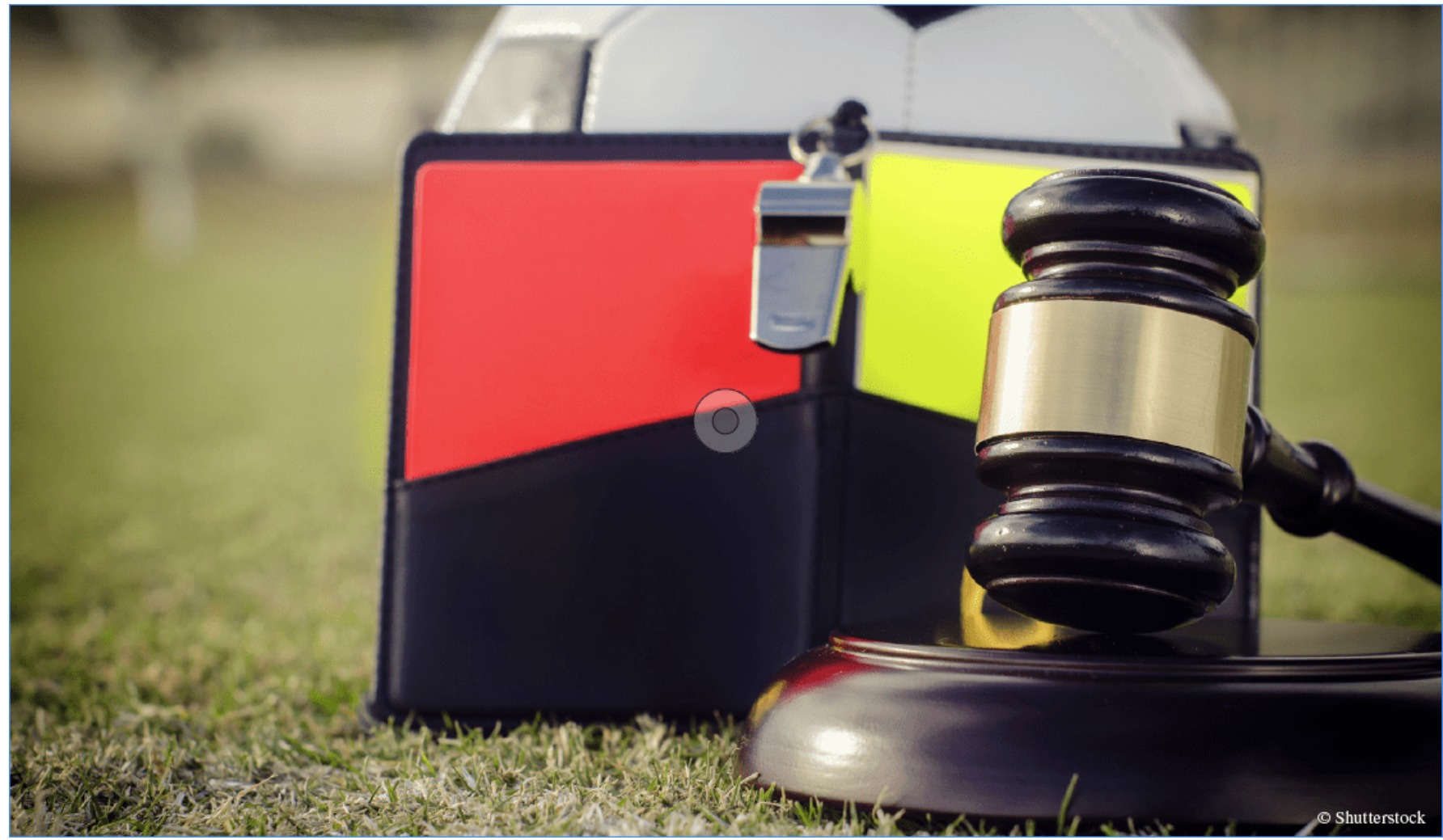

\section{Bazzi Claudio}

Partner/Co-Head Industry Group Sports, Bratschi AG, Zürich

\begin{abstract}
From a legal point of view, sports medical team care is characterized by various areas of tension. In particular, sports physicians have to balance health protection with patient autonomy, professional secrecy with the protective rights of third parties, and enhancing physical performance with fair competition. These dilemmas occur in extremely compressed form in the case of head injuries during competition. Using this example for illustrative purposes, elements for the resolution of such dilemmas are discussed hereinafter which lead to the following conclusion: Provided that sports medicine personnel succeed in taking measures appropriate to the individual case, supported by adequate rules and
\end{abstract}


regulations, the various areas of tension appear to be manageable in practice and do not turn into a legal minefield.

\section{Zusammenfassung}

Die sportmedizinische Mannschaftsbetreuung ist aus rechtlicher Sicht von verschiedenen Spannungsfeldern geprägt. So haben Sportärzte namentlich den Gesundheitsschutz mit der Patientenautonomie, das Berufsgeheimnis mit den Schutzinteressen Dritter sowie die Leistungsförderung mit der Wettkampfethik auszubalancieren. Besonders komprimiert treten diese Dilemmata im Falle von Kopfverletzungen während eines Wettkampfs auf. Anhand dieses Illustrationsbeispiels werden im folgenden Beitrag Elemente zu deren Auflösung diskutiert. Er kommt zum Schluss, dass, sofern es dem sportmedizinischen Personal gelingt, unterstützt von adäquaten Regelwerken einzelfallgerechte Massnahmen zu treffen, die verschiedenen Spannungsfelder in der Praxis zu bewältigen sind und nicht in ein rechtliches Minenfeld umzuschlagen drohen.

\section{Einführung}

In Zeiten einer globalen Pandemie und deren Auswirkung auf den Mannschaftssport und Grossveranstaltungen ist die Gesundheit von Spitzensportlern wieder vermehrt in den Fokus der Öffentlichkeit gerückt. Während der Breitensport in Europa - wenigstens während der zweiten Welle der Covid-19-Krise - weitgehend lahmgelegt wurde, laufen die Meisterschaften der wichtigsten Mannschaftssportarten auf nationaler und internationaler Ebene weiterhin auf Hochtouren. Möglich geworden ist dies nicht zuletzt dank in der Gesellschaft breit abgestützten Schutzkonzepten. Dennoch besteht gerade bei Vollkontaktsportarten selbstredend eine erhöhte Virusübertragungsgefahr, wodurch der Ausfall von Spielern und ganzen Mannschaften droht. Exemplarisch zeugt davon die im Januar 2021 in Ägypten durchgeführte Handball-WM, bei der mehrere Mannschaften kurz vor oder während dem Turnier ihren Rückzug erklären mussten.

Mit der gesteigerten Gefährdungslage durch die Pandemie hat sich auch die - ohnehin schon erhebliche Verantwortung von medizinischem Fachpersonal, in erster Linie von Sportärzten, aber etwa auch von Physiotherapeuten (welche im Folgenden als miteingeschlossen gelten), für die Gesundheit der von ihnen betreuten Spitzensportler noch einmal erhöht. Grund genug also, um die wichtigsten rechtlichen Leitplanken der medizinischen Athletenbetreuung, insbesondere im Rahmen von Mannschaftssportarten, in Erinnerung zu rufen und anhand eines konkreten, häufig vorkommenden Verletzungsmusters zu illustrieren.

\section{Rechtliche Spannungsfelder bei der sportmedizinischen Mannschaftsbetreuung}

Der Berufsalltag von Sportärzten war - schon vor Ausbruch der Covid-19-Pandemie - und ist heute erst recht von einer komplexen Gemengelage zwischen Aspekten von Sport, Medizin und Recht geprägt, welche Praktiker immer wieder zu heiklen Abgrenzungs- und Ausscheidungsentscheidungen zwingt. Die wichtigsten, nachstehend kurz zu erörternden Spannungsfelder, verstanden als eine Mehrzahl von sich (potenziell) widersprechenden rechtlichen Pflichten, sind: 
1. Gesundheitsschutz vs. Patientenautonomie

2. Berufsgeheimnis vs. Drittinteressen

3. Leistungsförderung vs. Berufs- und Wettkampfethik

\section{Gesundheitsschutz vs. Patientenautonomie}

Der wichtigste Grundsatz der medizinischen Betreuung, und zugleich oberste rechtliche Pflicht, nämlich den Gesundheitsschutz des Patienten zu gewährleisten, besitzt selbstverständlich auch im Kontext des Mannschaftsspitzensports Gültigkeit. Allerdings ist die Interessenlage oft einiges vielschichtiger als bei der gewöhnlichen Arzt-Patient-Beziehung: So steht der Sportler unter erheblichem Leistungsdruck, welcher nicht nur selbst, sondern auch durch Dritte, namentlich durch seinen Verein bzw. Verband (und damit Arbeitgeber), sowie womöglich durch die Öffentlichkeit, auferlegt ist [1]. Der inhärente potenzielle Zielkonflikt - ein gesundheitlich gefährdeter, aber wettkampfwilliger Athlet - stellt Sportmediziner vor schwierige Abwägungsfragen. So müssen sie, gegebenenfalls im Wettkampf selbst unter erheblichem Zeitdruck, entscheiden, ob unter Berücksichtigung aller relevanten Parameter des Einzelfalls (Grad des gesundheitlichen Risikos, Entscheidungsfähigkeit des Betroffenen, Gefährdung Dritter usw.) dem Wunsch des Sportlers stattgegeben und eine Wettkampftauglichkeit attestiert werden kann oder nicht. Die Covid-19-Pandemie hat diesem Prozess eine zusätzliche Komplikationsstufe hinzugefügt, indem unter Umständen auch die (indirekten) Auswirkungen eines Wettkampfeinsatzes auf die Gesamtgesellschaft in die Überlegungen miteinzubeziehen sind. Äusserste Grenze der ärztlichen Entscheidungsfreiheit bildet jedenfalls das Gewissen: Es dürfen keine Empfehlungen abgegebenen werden, die mit selbigem schlechterdings nicht mehr zu vereinbaren sind [2].

\section{Berufsgeheimnis vs. Drittinteressen}

Die Schweigepflicht von Ärzten gehört - angesichts der notwendigen Einsichtnahme in höchstpersönliche Sachverhalte - zu den Fundamentalprinzipien der Berufsausübung. Ein Verstoss dagegen wird strafrechtlich sanktioniert [3]. In der Praxis kann es jedoch zu Situationen kommen, in denen Dritte zu deren eigenen Schutz über den Gesundheitszustand des betreuten Athleten zu informieren sind. Sofern der betroffene Sportler dazu kein Einverständnis gibt oder geben kann, muss der Arzt sich von der zuständigen kantonalen Behörde vom Berufsgeheimnis entbinden lassen [4]. Leitet er Informationen ohne Einwilligung des Sportlers oder der Behörde weiter, riskiert er eine strafrechtliche Verfolgung. Eine Ausnahme erfährt dieser Grundsatz nur dort, wo eine Verletzung der Schweigepflicht notwendig ist, um höherwertige Interessen (etwa die Gesundheit Dritter) zu schützen. Die Hürden für den sogenannten rechtfertigenden Notstand sind jedoch hoch, und es sollte entsprechende Zurückhaltung geübt werden [5]. Das beschriebene Spannungsverhältnis hat sich mit der Covid-19-Pandemie zweifelsohne verstärkt, da bei Ansteckungen die zeitnahe Information von potenziell gefährdeten Dritten (im Falle von Mannschaftssportarten regelmässig eine grosse Anzahl von Spielern) zur staatlichen Eindämmungsstrategie zählt. Gleichzeitig hat eine Verschiebung in Richtung erhöhter Informationsdurchlässigkeit von Gesundheitsdaten stattgefunden, und für den Persönlichkeitsschutz der Betroffenen bleibt wenig Raum [6]. 
Einerseits entspricht es dem Kernauftrag von sportmedizinischen Betreuern, ihre Athleten hinsichtlich der Steigerung der körperlichen Leistungsfähigkeit zu unterstützen. Andererseits tragen sie eine hohe ethische Verantwortung: Zunächst dem eigenen Berufsstand gegenüber, welcher keine Praktiken zulässt, die im Widerspruch zum eigenen Gewissen stehen (siehe oben). Ebenso aber eine für die Glaubwürdigkeit des Sports als Ganzem, nämlich einen fairen Wettbewerb zu gewährleisten. Und nicht zuletzt besteht eine Verpflichtung zum Schutz der Gesundheit des Sportlers (siehe oben). Aus diesen Grenzen des Leistungsförderungsgebots des Sportmediziners leitet sich ein - sowohl auf staatlicher Ebene als auch im sportautonomen Regelungsbereich mittlerweile breit abgestütztes - Verbot des Einsatzes von Doping ab [7]. Offensichtlicher Missbrauch, etwa die Besorgung und Weitergabe von verbotenen Substanzen, liegt klarerweise ausserhalb der ärztlichen Entscheidungsfreiheit. Hingegen besteht die Notwendigkeit zur sorgfältigen Abwägung dort, wo leistungsfördernde Mittel verabreicht oder Behandlungsmethoden angewandt werden, welche zwar (noch) nicht verpönt sind, aber dennoch in einer unangemessenen Beeinflussung des Wettbewerbs oder einer Gefährdung des betreuten Athleten resultieren.

\section{Illustration anhand eines Verletzungsmusters: Kopfverletzungen}

Eines der drängendsten sportmedizinischen Probleme in Vollkontaktmannschaftssportarten sind Kopfverletzungen. So wurden in den vergangenen Jahren eine Vielzahl an Fällen von Langzeitschäden ehemaliger Football- und Eishockey-Spieler der US-amerikanischen Profiligen offenbar [8]. Als Reaktion hierauf führte etwa die National Football League (NFL) - neben der Leistung von Schadenersatzzahlungen - ein sogenanntes «Concussion Protocol» ein, also eine festgelegte Vorgehensweise, welche im Fall einer Kopfverletzung eine unverzügliche und adäquate Behandlung des betroffenen Spielers ermöglicht [9]. Damit soll insbesondere Kurz- und Langzeitfolgen von Gehirnerschütterungen entgegengewirkt werden. Doch nicht nur der US-amerikanische Sport ist vom Problem der Kopfverletzungen betroffen, auch der europäisch geprägte Profifussball hat selbiges erkannt. Exemplarisch dafür steht der Fall des deutschen Fussballers Christoph Kramer, welcher im Finale der Fussball-Weltmeisterschaft 2014 ein heftiges Tackling gegen den Kopf erlitt, nach kurzer Behandlungspause aber weiterspielte. Im Folgenden trat jedoch seine offensichtliche Spielunfähigkeit zu Tage, unter anderem in Form der an den Schiedsrichter gerichteten Frage, ob er sich tatsächlich im Weltmeisterschafts-Finale befinde [10]. Der Weltfussballverband (FIFA) reagierte darauf sowie auf ähnliche Vorfälle, indem er im Februar 2021 an der in Katar durchgeführten Klub-Weltmeisterschaft eine Regeländerung testete, welche eine zusätzliche Auswechslung im Fall einer (vermuteten) Gehirnerschütterung ermöglicht [11].

Die Diagnostik im Fall von Kopfverletzungen stellt medizinische Betreuer vor besondere Herausforderungen. Erstens, weil die Schwere der Verletzung oftmals äusserlich nicht ohne Weiteres sichtbar ist, sondern erst in einem systematischen Testverfahren eruiert werden kann. Zweitens, weil sich der betroffene Athlet im Wettkampfmodus befindet und sein Körper- und Schmerzempfinden - und damit auch seine Wahrnehmungskompetenz betreffend die eigene Gesundheit - gegebenenfalls eingeschränkt ist. Drittens, weil erheblicher Zeitdruck besteht, da der Mannschaft während der Behandlungspause ein Spieler fehlt und so ein entscheidender Nachteil erwachsen könnte. Viertens, weil der Ausfall eines (Schlüssel-)Spielers gravierende Auswirkungen auf den Spielverlauf haben könnte und der medizinischen Beurteilung folglich grosse Bedeutung zukommt. 
Unter diesen Prämissen treten alle die vorbeschriebenen rechtlichen Dilemmata in komprimierter - je nach Tragweite des Wettkampfs zusätzlich potenzierter - Form auf: Welches Gewicht misst der Arzt dem unter Umständen unbedingten Willen des Spielers zur Fortsetzung des Wettkampfs zu? Wer wird wann und in welcher Weise informiert über allfällige Verletzung und andere gesundheitliche Beeinträchtigungen? Sollen dem Spieler Medikamente verabreicht werden, welche ihm das Weiterspielen ermöglichen oder nicht?

Eine generelle Blaupause, wie in solchen Fällen zu verfahren ist, gibt es nicht. Sowohl aus medizinischer als auch aus rechtlicher Sicht ist jeweils eine einzelfallbasierte Empfehlung unter Berücksichtigung sämtlicher relevanter Umstände abzugeben. Das Bewusstsein des Sportmediziners für die Bedeutung des Einzelfalls und die damit verbundene Verantwortung ist elementar, damit er in der Hitze des Gefechts richtig funktionieren kann. Weiter sollte er sich der - oben besprochenen - relevanten rechtlichen Spannungsfelder, innerhalb derer er sich bewegt, bewusst sein. Je besser diese im Gedächtnis abgespeichert sind, desto einfacher sind sie im Ernstfall auch abruf- und anwendbar. Schliesslich sollte sich der behandelnde Arzt unbedingt die für eine sorgfältige Diagnostik notwendige Zeit ausbedingen. Eine eindeutige, vorgefasste Gewichtung der verschiedenen widerstreitenden Elemente scheint hingegen nicht angezeigt: Zwar liegt es in der Natur des Ärzteberufs, die Gesundheit der Patienten - zuweilen auch gegen den äusseren Leistungsdruck - zu verteidigen. Allerdings ist Spitzensport per se mit gesundheitlichen Risiken verbunden. Insofern würde sich medizinisches Personal, welches einseitig auf Gesundheitsschutz setzt, von vornherein in einen unauflösbaren Widerspruch begeben (und faktisch auch nicht allzu lange das Vertrauen von Vereinen bzw. Verbänden geniessen).

Letztlich liegt es jedoch auch in der Verantwortung von rechtssetzenden Instanzen, sei es auf staatlicher oder auf sportautonomer Ebene, die richtigen Rahmenbedingungen für das sportmedizinische Personal zu schaffen. Vor diesem Hintergrund sind Bestrebungen wie die vorerwähnten der NFL oder der FIFA zu begrüssen, welche den Druck auf Sportärzte, sofort (möglicherweise spielentscheidende) Einschätzungen liefern zu müssen, mindern können. Zwei Faktoren sind dabei von Bedeutung: Erstens der zeitliche Aspekt. Gerade Kopfverletzungen verlangen nach einer eingehenden Untersuchung des Spielers, welche unter Zeitdruck zuweilen entscheidender Elemente verlustig geht. Zweitens die Neutralisierung von spielstrategischen Nachteilen. Die von der FIFA angedachte Möglichkeit einer zusätzlichen Wechselmöglichkeit im Falle von Kopfverletzungen senkt den impliziten Druck, einen Spieler trotz Verletzung weiterspielen zu lassen, erheblich.

\section{Fazit}

Die sportmedizinische Praxis ist zwar tatsächlich geprägt von rechtlichen Spannungsfeldern, welche sich durch die spezifischen Herausforderungen der Covid-19-Pandemie noch verstärkt haben dürften. Sofern die damit verbundenen Abwägungen im Verletzungsfall sorgfältig und unter Berücksichtigung der relevanten Entscheidungsparameter getroffen werden, sind diese Spannungsfelder jedoch zu bewältigen, ohne dass wesentlich erhöhte zivil- und strafrechtliche Haftungsrisiken bestehen. Von einem rechtlichen Minenfeld kann bei sorgfältiger Vorgehensweise folglich nicht die Rede sein. 


\section{Acknowledgments, conflict of interest and funding} None declared.

\section{Corresponding author}

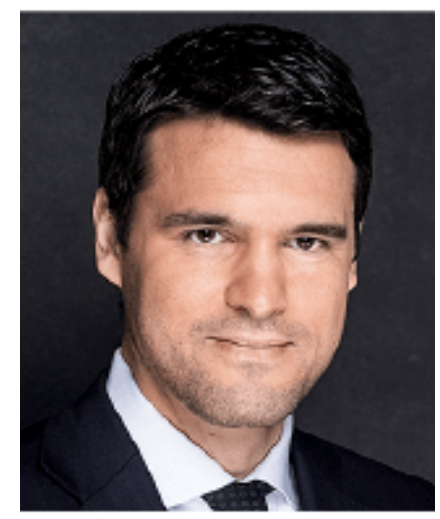

\section{Claudio Bazzi}

Bahnhofstrasse 70, 8001 Zürich

Tel. 0582581000

E-Mail: claudio.bazzi@bratschi.ch 


\section{CONCUSSION GAME DAY CHECKLIST}

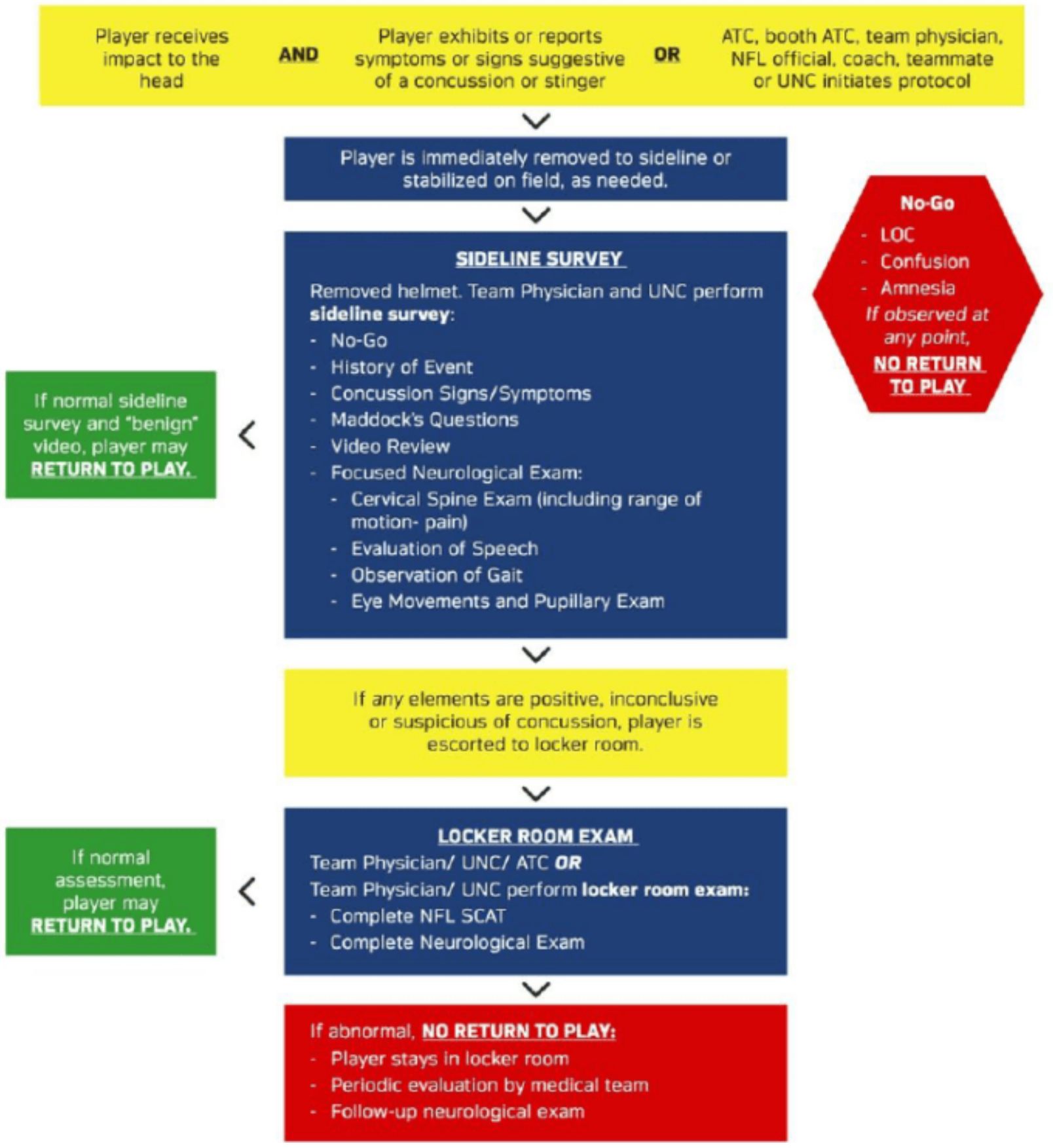

\section{References}

1. Anhang 5 zur Standesordnung FMH / Richtlinien für die ärztliche Betreuung von Sporttreibenden, revidierte Version vom 19. Dezember 2018, Ziff. 3.1 
2. Standesordnung der FMH, revidierte Version vom 31. Oktober 2019, Art. 3 Abs. 4

3. Schweizerisches Strafgesetzbuch vom 21. Dezember 1937 (Stand

4. Juli 2020), Art. 321

5. Im Kanton Zürich ist dafür etwa die Gesundheitsdirektion / Abteilung Recht zuständig, vgl. https://www.zh.ch/de/gesundheit/gesundheitsberufe.html\#-993151727 (zuletzt abgerufen am 14. Februar 2021)

6. Alexander Marcel Niggli / Carola Göhlich, Basler Kommentar zum Strafrecht, Art. 17 N 1 ff., 4. Auflage, Basel 2019

7. Vgl. für den arbeitsrechtlichen Kontext: Thomas Geiser / Roland Müller / Kurt Pärli, Klärung arbeitsrechtlicher Fragen im Zusammenhang mit dem Coronavirus, in: Jusletter 23. März 2020

8. 7 Für die Schweiz: Bundesgesetz über die Förderung von Sport und Bewegung vom 17. Juni 2011 (Stand 1. Januar 2021), insbesondere Art. 19 und 22; Exemplarisch für die internationale Sportwelt: Der Welt-Anti-Doping-Code der Welt-Anti-Doping-Agentur (WADA), einsehbar unter https://www.wada-ama.org/sites/default/files/resources/files/2021_wada_code.pdf (zuletzt abgerufen am 14. Februar 2021

9. Beispielhaft dazu «Gehirnerschütterung: Fatale Beschleunigung», Zeit online, 23. Mai 2013, einsehbar unter https://www.zeit.de/2013/22/gehirnerschuetterung-folgen-sport (zuletzt abgerufen am 14.

Februar 2021)

10. Details sind einsehbar unter https://www.nfl.com/playerhealthandsafety/health-and-wellness/player-care/concussion-protocol-ret urn- to-participation-protocol (zuletzt abgerufen am 14. Februar 2021)

11. «Christoph Kramer: Der Weltmeister, der das Finale vergass», Sueddeutsche Zeitung online, 25. Mai 2018, einsehbar unter

https://www.sueddeutsche.de/sport/fussball-christoph-kramer-der-weltmeister-der-das-finale-vergass -dpa.urn-newsml-dpa-com-20090101-180525-

12. 99-447766 (zuletzt besucht am 14. Februar 2021)

13. «Fifa testet zusätzliche Wechsel bei Gehirnerschütterungen», Spiegel online, 8. Januar 2021, einsehbar unter https://www.spiegel.de/sport/fussball/gehirnerschuetterung-im-fussball-fifa-testet-zusaetzliche-wechs el-bei-klub-wm-a-54b10a23-7b65-497f-95fb-288973451eae (zuletzt abgerufen am 14. Februar 2021) 